\title{
Oesophageal tuberculosis: a review of eleven cases
}

\author{
T. Mokoena' ${ }^{1}$ D.M. Shama ${ }^{2}$, H. Ngakane' and J.V. Bryer ${ }^{1}$ \\ Departments of ${ }^{1}$ General Surgery and ${ }^{2}$ Thoracic Surgery, University of Natal Medical School, Durban, \\ Republic of South Africa
}

\begin{abstract}
Summary: Tuberculous infection of the oesophagus is rare. This is confirmed by our present review of cases managed in our teaching hospitals over a period of 18 years which uncovered only 11 patients. The main presentation is that of dysphagia whose algorithm of investigation should seek to differentiate tuberculosis from carcinoma, the more common cause of this symptom. Of the 11 patients, 9 presented with dysphagia while 2 had haemorrhage; 7 had an abnormal plain chest radiograph, of whom 4 had a mediastinal mass lesion ( 3 were lymphadenopathy and one an abscess). All but one had an abnormal radio-contrast oesophagogram, including a mediastinal sinus in two and a traction diverticulum in another two. The mainstay of investigation was oesophagoscopy through which diagnostic biopsy material was obtained in half of the patients. In the other half diagnosis was by either biopsy of associated mediastinal (3) or cervical (1) lymph node masses or by acid fast bacilli positive sputum (1). The diagnosis was established post-mortem in one patient.

Treatment was primarily non-operative with standard anti-tuberculosis drug therapy. Two patients underwent a diagnostic thoracotomy and one a drainage of mediastinal abscess together with resection and repair of oesophago-mediastinal sinus during the early part of the series. Outcome of management was very rewarding in 9 patients and death occurred in 2 patients, one of whom had his anti-tuberculosis drug therapy interrupted by severe hepatitis B virus infection. The other death occurred in a patient whose haemorrhage from an aorta-oesophageal fistula was not established ante-mortem. It is recommended that when biopsy material of the oesophagus is unobtainable or non-diagnostic in patients with dysphagia, especially with an abnormal chest radiograph or human immunodeficiency virus infection, effort should be made to obtain biopsy material from associated lymph nodes, even by thoracotomy if necessary, or culture of biopsy from the radiologically abnormal part of oesophagus and sputum for mycobacteria, in order to establish the diagnosis of this rare but eminently treatable cause of dysphagia. Clinicians should be aware of tuberculosis of the oesophagus as a possible cause of haematemesis in patients with otherwise unexplained upper gastrointestinal haemorrhage.
\end{abstract}

\section{Introduction}

Tuberculosis (TB) of the oesophagus is rare. ${ }^{1,2}$ When present, it is usually secondary to current or previous tuberculous infection in other anatomical sites. Most reported cases have been secondary to respiratory tract infection. ${ }^{1-5}$ Some have shown involvement of the thoracic aorta. ${ }^{6,7}$ The principal presentation has been dysphagia but many had pain $^{1-5}$ and few presented with haematemesis. ${ }^{6,7}$ Because of the rarity of TB as a cause of symptomatic oesophageal disease, the diagnosis has often been missed as diagnostic efforts were directed at the more common causes such as carcinoma and peptic ulceration and, in cases of

Correspondence: T. Mokoena, Department of Surgery, Faculty of Medicine, University of Natal, PO Box 17039, Congella 4013, Durban, RSA.

Accepted: 27 September 1991 haemorrhage, at oesophageal varices. However, unlike these other diseases, TB is eminently curable and therefore it is imperative that clinicians should bear it in mind when managing patients with oesophageal disease symptoms. Although TB oesophagus is rare worldwide, it is not confined to developing countries where TB is endemic, but is being seen increasingly in developed countries with significant immigrant populations and would be expected in native populations with the increase of acquired immunodeficiency syndrome (AIDS) which is often complicated by mycobacterial infections. TB is prevalent in our environment and therefore it would be expected that the oesophageal disease would be relatively less uncommon. A review of records of patients with TB of the oesophagus was undertaken in order to uncover patterns of presentation and outcome in management of this disease at the teaching hospitals of the University of Natal Medical School. 


\section{Material and methods}

Available case records of those patients who have been diagnosed as having TB with oesophageal involvement from 1972 to 1990 were studied.

\section{Results}

There were 11 patients whose records were sufficiently complete to permit a suitable analysis to be carried out (Table I). There were 6 male and 5 female patients whose mean ( \pm s.d.) age was 37.9 ( \pm 14.7 ) years. The main presenting symptom was dysphagia in 9 patients. Two patients presented with haematemesis. Hoarseness of the voice was present in 2 patients. A plain chest radiograph was abnormal in 7 patients $(64 \%)$, where 4 had paratracheal or mediastinal mass lesions, one of which turned out to be a tuberculous abscess at operative exploration and one each had pleural effusion and extensive old fibrosing pulmonary lesion.

An oesophagogram showed external compression in 4 patients ( $36 \%$ ) and these were the patients with mass lesions of the mediastinum (Figure 1). A mediastinal sinus was demonstrated in $2(18 \%)$, a traction diverticulum in 2, and in one each a polypoid tumour, an ulcer (Figure 2) and an irregularity of the mucosa. One patient with proximal oesophageal TB had a normal oesophagogram.

All patients underwent oesophagoscopy. This confirmed the oesophagogram findings of a sinus in 2 patients, a traction diverticulum in one, a polypoid tumour in another and uncovered an ulcerating mass in a patient whose oesophagogram merely showed extrinsic compression (patient no. 5, Table I). It also demonstrated the ulcer in one of the haematemesis patients as well as showing proximal involvement of the oesophagus in a patient with laryngeal TB. It failed to reveal the aorta-oesophageal fistula in the other haematemesis patient whose clinical details have been reported previously. $^{\text {? }}$

The definitive diagnosis of TB was made on histological demonstration of the classical caseous TB granuloma or acid fast bacilli in 8 patients $(72 \%)$, on clinical grounds in 2 patients and at autopsy in one. Three patients who were attended to during the early 1970 s period were managed operatively for procurement of mediastinal lymph node biopsy in 2 and drainage of a mediastinal abscess in one who also had resection of an oesophageal sinus and repair of the oesophagus. All patients were managed by standard anti-TB drug treatment.

There were 2 deaths, one in a patient whose effective anti-TB therapy was interrupted by type B viral hepatitis with jaundice and convulsions. This patient also had severe cor pulmonale and he died 8 months after diagnosis. The other death occurred in a patient with a missed aorta-oesophageal fistula who had a fatal haemorrhage after two weeks of hospitalisation and a fruitless gastroduodenal exploration, vagotomy and pyloroplasty. The other 9 patients were well at the last time of medical attendance which varied from three months to 12 years.

\section{Discussion}

Tuberculosis is capable of mimicking many disease processes and may affect any anatomical structure. Whereas in developing countries where tuberculosis is common the diagnosis is often made, in the more sophisticated countries TB may not be considered in a primary list of differential diagnoses.

Although the oesophagus is a rare site for TB to cause symptoms, the problem is well recognized, as attested to by many sporadic case reports in the literature. ${ }^{1-5}$ Our experience with 11 patients spanning a period of 18 years further illustrates the low frequency of this disease in an environment in which TB is otherwise endemic.

The most common presenting symptom is dysphagia, and haematemesis the least common. Occassionally patients present with oesophagobronchial fistulae whose symptoms include coughing after ingestion of liquids. ${ }^{3}$ These symptoms are similar to those produced by carcinoma of the oesophagus which is much more prevalent. ${ }^{2}$ The approach to their clinical investigation therefore follows the same guidelines used in carcinoma of the oesophagus and, among others, seeks to obtain tissue diagnosis.

A plain chest radiograph is taken as part of the routine work for patients with dysphagia. If this shows abnormality, especially if a mediastinal mass or an apical lesion suggestive of present or past TB is noted, the possibility of oesophageal TB should be strongly suspected. However, it is common to note mediastinal lymphadenopathy in patients with pulmonary TB but only rarely is this associated with dysphagia. A rare feature seen on chest $\mathrm{X}$-ray is a mediastinal abscess which results from breakdown of TB lymph nodes forming a 'cold' abscess or less frequently from a breach of the oesophageal wall integrity and contamination by pyogenic organisms. All the plain radiograph changes associated with TB may be seen in carcinoma of the oesophagus although pulmonary changes in carcinoma tend to affect the base of the lungs due to repeated aspiration. However a caveat that the two diseases may co-exist needs to be added and this should be kept in mind especially in our environment where both are endemic.

A radio-contrast oesophagogram is obtained as 


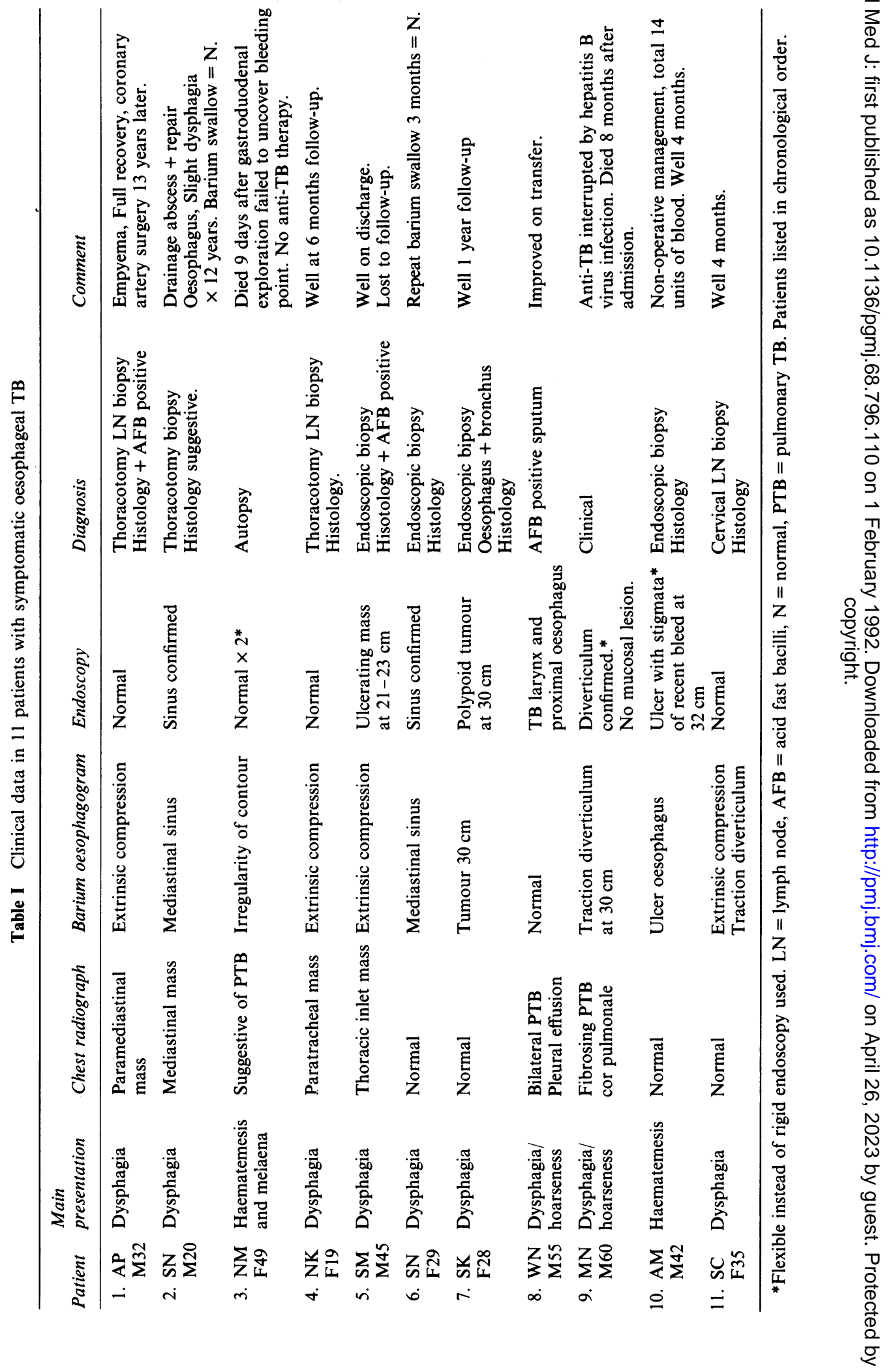




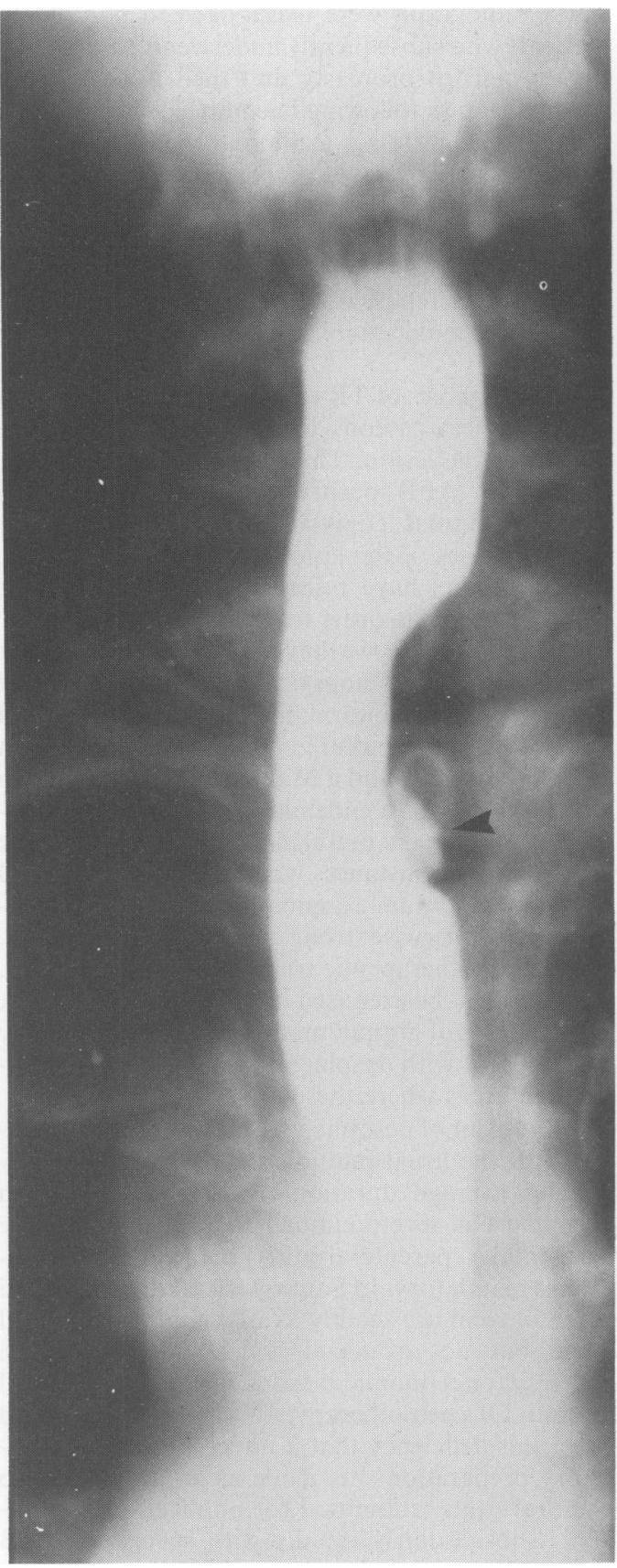

Figure 1 An illustrative barium oesophagogram of a patient presenting with dysphagia shows the frequent external compression of the oesophagus by enlarged medisatinal lymph nodes. Note a small diverticulum into the compressing mass (arrow). This patient had a 'normal' oesophagoscopic examination but biopsy of her cervical lymph nodes which were also enlarged proved to be TB. She responded well to anti-TB treatment.

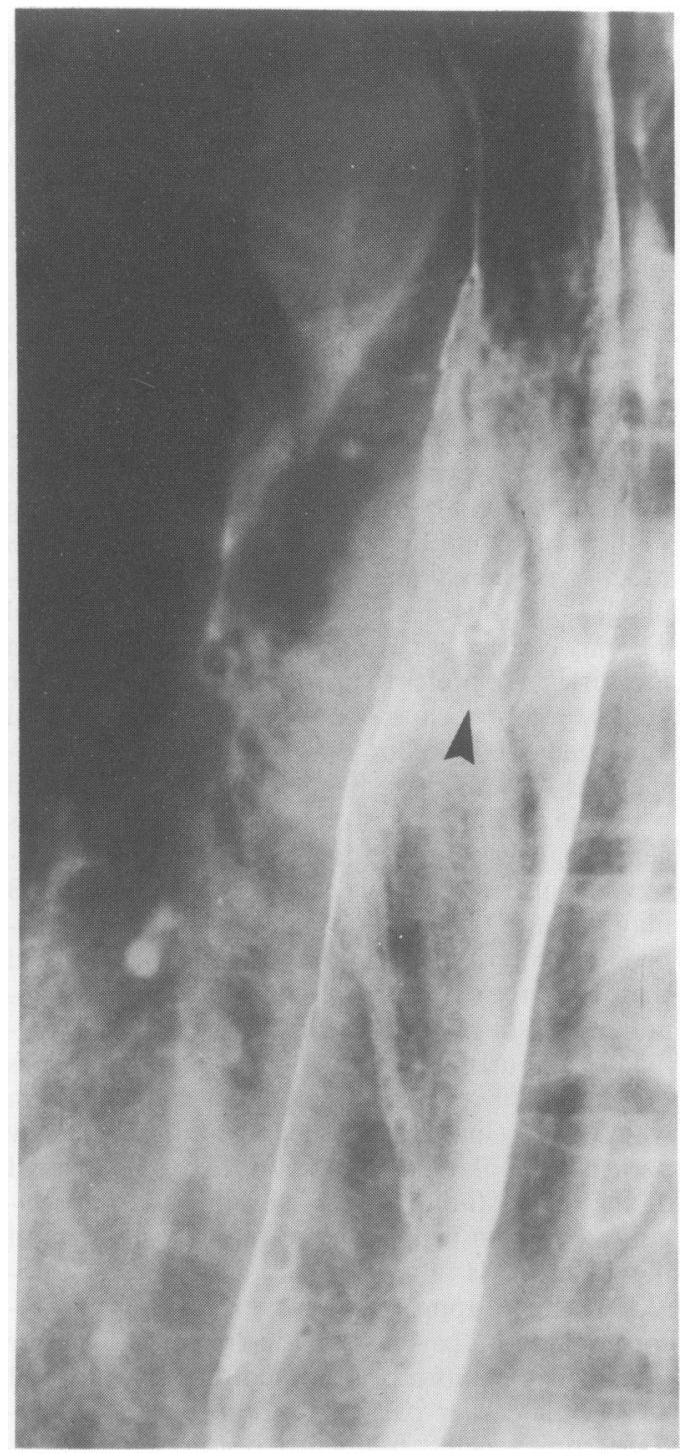

Figure 2 A barium oesophagogram of a patient presenting with haematemesis and melaena demonstrates an ulcerating lesion (arrow) which on oesophagoscopic examination showed stigmata of recent bleeding. Biopsy of the lesion showed TB.

a prelude to oesophagoscopy. The most common feature is extrinsic compression of the oesophagus usually by a mass of tuberculous lymph nodes. Although the oesophageal mucosa is often intact in such cases, the adventitia is usually implicated and this may produce a classical traction diverticulum demonstrated on the oesophagogram (Figure 1). When the process involves the oesophageal mucosa an exuberant tumorous lesion or an ulcer may -esult, both of which are radiographically indistin- 
guishable from carcinoma. The contrast oesophagogram may demonstrate a sinus into the mediastinum or a fistula into the tracheobronchial tree. Finally a constrictive lesion may result which may be radiographically indistinguishable from the more common causes of benign oesophageal structure. ${ }^{1,3}$ This latter presentation was not seen in our 11 cases.

An oesophageal endoscopy is the key investigation in evaluation of dysphagia for procurement of biopsy material. The preliminary radio-contrast oesophagogram assists the endoscopists in directing particular attention to the area of interest. It is our practice to perform a tracheo-bronchoscopy as well in all patients with dysphagia as part of staging of the more common oesophageal carcinoma. This practice is well suited to cases of suspected oesophageal TB as well since endotracheal or laryngeal TB may coexist, ${ }^{8}$ as was illustrated by 2 patients in this series. We prefer to use a rigid oesophagoscope and a rigid bronchoscope which require a general anaesthetic. Unlike some workers ${ }^{9}$ a flexible endoscope is only used on haematemesis patients and when the general condition of the patient precludes general anaesthetics. There are no specific endoscopic features upon which a firm diagnosis of TB may be rested. Indeed in many patients the oesophageal mucosa remains macroscopically clear and therefore the requisite biopsy material unobtainable as random mucosal biopsy is not recommended. Fortunately these patients sometimes present with cervical lymphadenopathy, from which biopsy material should be obtained. In a few patients the enlarged lymph nodes are only located in the mediastinum where they may be indistinguishable from mediastinal tumours or cysts and the diagnosis is then only made after mediastinoscopy, where appropriate, or exploratory thoracotomy and biopsy. This is illustrated by 3 of our cases where exploratory thoracotomy was utilized during the earlier part of the series when mediastinoscopy was less frequently performed - nowadays mediastinoscopic lymph node biopsy would have been offered before exploratory surgery unless a cyst or an abscess was very strongly suspected.

An especially challenging presentation is that of haematemesis where TB of the oesophagus is unlikely to be part of the initial differential diagnosis. In these patients flexible endoscopy is usually the first investigation and when the usual causes and sites of upper gastrointestinal tract bleeding are not demonstrated a more careful oesophageal examination is necessary. It might be necessary to resort to rigid endoscopy. An abnormal chest radiograph should alert the clinician to the possibility of TB of the oesophagus as the cause of haemorrhage; this clue was missed in the case reported by Hancock and Barnett. ${ }^{6}$ An early oesophagogram might also give a clue to the diagnosis. Sadly, this and an abnormal chest radiograph were overlooked in one of our patients who subsequently underwent fruitless vagotomy and pyloroplasty and died 2 weeks after hospitalization following haemorrhage from aortaoesophageal fistula. ${ }^{7}$ Radio-contrast oesophagogram and endoscopy complement each other in investigation of proximal alimentary tract disease and therefore when one is negative the other should be used before the patient is dismissed. We have often had to repeat each one in quick succession before we could establish a diagnosis in difficult cases.

The diagnosis of TB rests on the demonstration of a classical caseous lesion or acid fast bacilli (AFB) in the lesion. This is also made where the sputum is AFB positive and the oesophageal biopsy material equivocal, but excludes other known causes of the lesion, especially carcinoma. Some authors have resorted to culturing of the biopsy material in order to establish the diagnosis. Although to date we have not done this in our practice, a repeat biopsy taken from the area of oesophagogram abnormality should be submitted for mycobacteria culture in diagnostically difficult cases. We have found a Mantoux test unreliable in diagnosis of TB in endemic areas like ours. However, this remains a useful indicator in non-endemic areas. In a few instances where neither biopsy, TB culture nor sputum examination succeeds in estab? lishing an otherwise strong clinical suspicion of TB a successful therapeutic trial often does. However, caution must be exercised in order not to overlook other causes of granulomatous diseases which may also present with dysphagia (such as histoplasmo$\left.\operatorname{sis}^{10}\right)$ in areas where these are endemic.

Treatment of oesophageal TB is primarily medical with the usual multiple anti-TB drug therapy for an average duration of 12 months. When dysphagia is severe enough to preclude effective oral intake, parenteral anti-TB drug administration is mandatory. In South Africa, rifampicin and streptomycin are readily available in a parentera form but not isoniazid which was withdrawn recently for economic reasons. Isoniazid is pivotal in anti-TB chemotherapy. It was in the effort to meet this deficiency that a novel isoniazid suppository preparation was made as an alternative to the oral route (submitted for publication). Symptoms subside fairly rapidly with specific anti-TB drug therapy and the oesophagogram reverts to normal within 3 months of treatment in most cases. Even the patients with sinuses heal well, with often a small diverticulum as the only remaining evidence of previous disease. However, this apparent rapid resolution should not tempt the clinician or the patient to cut short the duration of full therapy. The outcome of medical treatment is very rewarding. Of the 10 patients who received anti-TB treatment, 9 had an uneventful recovery. Death 
occurred in a patient whose treatment was interrupted by severe hepatitis B viral infection.

Surgical intervention is reserved for those patients in whom the diagnosis cannot be reached by endoscopy or biopsy of accessible associated lymph nodes such as mediastinal masses not suitable for mediastinoscopy and in those patients in whom complications demand surgery, for example a mediastinal abscess seen to be unresponsive to non-operative management. Although radical resection has sometimes been inadvertently performed, ${ }^{1}$ planned resection has been infrequent. ${ }^{8,11}$ The oesophageal lesion was tackled directly in only one case in this series. This was a patient in whom a mediastinal sinus had lead to abscess formation who underwent surgical drainage of the abscess together with successful resection of the sinus and oesophageal repair.

Although total dysphagia is uncommon, the usual strategies for nutritional support should be employed until swallowing improves with anti-TB drug therapy. We have recently used short to medium term total parenteral nutrition in selected cases of dysphagia, but a feeding gastrostomy is a suitable and more widely available alternative. It is in this type of case that we would recommend the use of our recently described INH suppositories (submitted for publication) together with parenteral streptomycin and rifampicin during the period of total parenteral nutrition. While dysphagia usually resolves with effective anti-TB treatment and nutritional support, troublesome stricture formation has occasionally led to radical resection and colon interposition. ${ }^{8}$

In summary, that oesophageal tuberculosis is rare has been borne out by this study which uncovered only 11 patients over a period of 18 years in an environment in which TB in general is still highly prevalent. However, with worldwide increase in human immunodeficiency virus (HIV) infection, which is often complicated by mycobacterial infections, its prevalence is expected to increase likewise. The main presenting symptom was dysphagia and as such the patients were subjected to an algorithm used for the more common carcinoma of the oesophagus. This is a suitable course as both rely on tissue biopsy for diagnosis. If endoscopic material is not obtainable then associated lymph nodes may provide the diagnostic material. When biopsy is not histologically diagnostic, material from areas of oesophagogram abnormality should be cultured for mycobacteria. Rarely, patients may present with haemorrhage where the algorithm followed is that of upper gastrointestinal bleeding. When gastrointestinal tract endoscopy fails to establish the more common sites and causes of bleeding, a radio-contrast oesophagogram should be considered. The plain chest radiograph is often abnormal and this should serve to alert the clinician to the diagnosis of TB, as should the presence of HIV infection. Once diagnosed, treatment relies firmly on anti-TB drug action, with surgery reserved for unresolving complications.

\section{References}

1. Fahmy, A.R., Guindi, R. \& Farid, A. Tuberculosis of the oesophagus. Thorax 1969, 24: 254-256.

2. Damtew, B., Frengley, D., Wolinsky, E. \& Spagnuolo, P.J. Esophageal tuberculosis: mimicry of gastrointestinal malignancy. Rev Inf Dis 1987, 9: 140-146.

3. Dow, C.J. Oesophageal tuberculosis: Four cases. Gut 1981, 22: $234-236$

4. Savage, P.E. \& Grundy, A. Oesophageal tuberculosis: an unusual cause of dysphagia. Br J Radiol 1984, 57: 1153-1155.

5. McNamara, M., Williams, C.E., Brown, T.S. \& Gopichandran, T.D. Tuberculosis affecting the oesophagus. Clin Radiol 1987, 38: 419-422.

6. Hancock, B.W. \& Barnett, D.B. Case of post-primary tuberculosis and massive haematemesis. $\mathrm{Br}$ Med J 1974, 3: $722-723$.

7. Robbs, J.V. \& Baker, L.W. Tuberculosis involving major arteries. J R Coll Surg Edinb 1976, 21: 353-358.

8. Montes, I., Larsen, E., Haiderer, O. \& Kennedy, J.H. Tuberculous stricture of the oesophagus: report of a patient successfully treated by colon interposition. Chest 1971, 60: 194-195.

9. Gordon, A.H. \& Marshall, J.B. Oesophageal tuberculosis: definitive diagnosis by endoscopy. Am J Gastroenterol 1990, 85: $174-177$

10. Wain, J.C. Jr \& Mark, E.J. A 48-year-old man with dysphagia, chest pain, fever and subcarinal mass. $N$ Engl $J$ Med 1991, 324: 1049-1056.

11. Sinha, S.N., Tesar, P., Seta, W. \& Sengupta, S.K. Primary oesophageal tuberculosis. Br J Clin Pract 1988, 42: 391 - 394. 